\title{
Ship roll stabilization control with low speed loss
}

\author{
Zhiquan Liu, Hongzhang Jin \\ College of Automation \\ Harbin Engineering University \\ Harbin, China \\ liuzhiquan215@sina.com
}

\author{
Michael John Grimble, Reza Katebi \\ Industrial Control Centre \\ Department of Electronic and Electrical Engineering \\ University of Strathclyde \\ Glasgow, UK
}

\begin{abstract}
Large roll motion induced by waves can severely affect the ability of vessels and the speed will loss due to added resistance which caused by ship motions, especially in moderate to high sea states. With increasing needs of fuel efficiency and greenhouse gas (GHG) emissions, the effect of added resistance on surface ship performance must be considered when a ship fin stabilizer control system is designed. In this paper, we investigate basic principles of added resistance in oblique waves and ship calm water resistance. An alternative approach for reducing speed loss while keeping the satify roll reduction percentage, is proposed by controlling both roll and roll rate at the same time. A double nonlinear generalized minimum variance (NGMV) controller is used for achieving this objective. Finally, the effectiveness of the method is demonstrated.
\end{abstract}

Keywords—added resistance; speed loss; fin stabilizer; NGMV

\section{INTRODUCTION}

Following the awareness of the increasing global warming and economic crisis, the ship speed loss and fuel consumption in actual weather condition is currently one of the main concerns for the ship building and ship industry, legislative actions have been taken on global and national levels [1]. Internationally, ship emissions are restricted by the International Maritime Organization (IMO), both the Energy Efficiency and Design Index (EEDI) and Energy Efficiency and Operational Index (EEOI) have also been considered by Marine Environment Protection Committee (MEPC) for the prevention of air pollution from ships [2]. Some researchers also began to target the speed loss. Prpić-Oršić and Faltinsen [1] developed a numerical model to predict the speed loss of a vessel in irregular sea. Chuang and Steen [3][4] used model tests and a time domain model to study the speed loss due to zig-zag motion in waves, then calculated the speed reduction of a vessel in oblique sea base on their former works. Fang etc. [5] investigated a simple alternative approach to assess the effect of the above-water bow form on the added resistance which would lead to speed loss for the ship advancing in longitudinal waves. Therefore, the energy efficency of voyage should be considered in ship motion control area.

As we know, a ship sailing in a seaway usually endured large motions and accelerations due to waves, which may lead to stability loss. At the same time, the total water resistance of a ship is increased by added resistance, resulting in speed reduction. It is reported that the magnitude of added resistance is about $10-30 \%$ of calm water resistance [6]. Ship motions, in particular, the vertical motions heave and pitch have the largest effect when calculating added resistance in head seas.
Nevertheless, the effect of roll motion on added resistance should be included because of the asymmetric modification of wave field which is made by the hull in the oblique sea case. The calculation of the impact of added resistance on yacht performance by Kai Graf et al. [7] presented that the maximum added resistance is affected only marginally by the presence of fins and sails. The forced rolling tests [8] in still water have shown that the mean resistance augmentation varies with the rolling amplitude. So, we confirm that the application of active fins can reduce added resistance in waves. Jin and Liu [9][10] have done some works about the relationship between ship rolling motion attitude and added resistance, and set up a rolladded-resistance model. But, there was not a very accurate method to settle three weighting factors in that model, only according to the calculation of hydrodynamic software. Liu and Jin [11] also intended to solve this problem from another view (i.e. the extention of radiated energy method proposed by Loukakis and Sclavounos [12]), an optimal added resistance roll stablilization control system was designed with the classical PID method, however without the description of speed. In this paper, we follow the former method to describe the specific speed loss due to ship roll motion. In the mean while, a double NGMV controller will be introduced to maintain the forward speed.

The paper is organized as follows. Section describes the theoretical calculation of added resistance, still water resistance and the speed loss caused by roll motion. Section briefly shows the system composition and controller design. Simulation results and discussion on the performance of the proposed control system are presented in Section Conclusions are drawn in Section .

\section{THEORETICAL CALCULATION}

Generally, the design propulsive power of a ship is based on still water resistance, and will be a constant when sailing on the open sea. So, the speed will be reduced for the increasing of total resistance (e.g. excited by wind, wave and current). In this paper, we only consider the effectiveness of resistance offered by wave, and the calculation is performed for full scale ship.

\section{A. Calm water resistance}

The resistance scaling method used in here follows the standard 1978 ITTC method. The method used to find the total resistance that the hull would be experienced by a "flat plate" equivalent surface area. 
The relationship between full scale total resistance $R_{T S}$ and the resistance coefficient $C_{T S}$ is

$$
R_{T S}=\frac{1}{2} \rho U^{2} S \times C_{T S}
$$

where $\rho$ is the water density, $U$ is the ship forward speed, $S$ is the area of ship wetted surface.

The total calm resistance coefficient can be computed using a friction line and form factor:

$$
C_{T S}=C_{R S}+\left(C_{F S}+\Delta C_{F S}\right)\left(1+k_{0}\right)
$$

$C_{R S}$ is the residual resistance coefficient, and $C_{R S}=$ $1.21 \times 10^{-3}$ according to the Taylor-Gertler method as there are no towing tank model test data can be adopted. $C_{F S}=0.075 /\left(\log _{10}^{\mathrm{Re}}-2\right)^{2}$ is the friction resistance coefficient, where Re is Reynolds number which is usually determined at $15^{\circ} \mathrm{C}$.

$$
\Delta C_{F S}=\left(110(H \times U)^{0.21}-403\right) \times C_{F S}^{2} \text { is a roughness }
$$
correction. $H=150\left(10^{-3} \mathrm{~mm}\right)$ is a standard value [4]. $k_{0}$ is form factor which is calculated with empirical equation $k_{0}=0.6 \varphi+145 \varphi^{3.5}$ and $\varphi=\left(C_{B} / L_{W L}\right) \sqrt{B_{W L}\left(T_{A}+T_{F}\right)}$, which $C_{B}$ is block coefficient, $L_{W L}$ is length of the water line, $B_{W L}$ is breadth of the water line, $T_{A}$ and $T_{F}$ are draught at aft perpendicular and forward perpendicular, respectively.

\section{B. Added resistance due to roll motion}

As shown in Fig. 1, a ship moving along $X$-axis direction. Two coordinate frames are considered: the first one $(\mathrm{X}, \mathrm{Y}, \mathrm{Z})$ is an inertial reference frame fixed with respect to the steady motion of ship, the second one $(\mathrm{x}, \mathrm{y}, \mathrm{z})$ fixed to the body motion which is not an inertial frame. A ship has 6 degree of freedom (DOF) motions with translation $\vec{\eta}_{T}=\left(\eta_{1}, \eta_{2}, \eta_{3}\right)$ and rotation $\vec{\eta}_{R}=\left(\eta_{4}, \eta_{5}, \eta_{6}\right)$ because of rigid-body. The added resistance is calculated in the inertial body reference frame. Here, $\beta$ is the encounter angle, $\beta=0$ if the waves are coming from the stern (i.e., following seas) and $\beta=\pi$ if the waves are coming from the bow (i.e., heading seas). $\phi$ is the rolling angle.

This average wave resistance increase is calculated by the method proposed by Loukakis and Sclavounos, and based on the extention of the method presented by Gerritsma and Beukelman [13] which is oringinally only for head sea. We will calculate the instantaneous added resistance in time domain follow the steps of Loukakis and Sclavounos.

The method of Gerritsma and Beukelman equates the work of the added resistance to the energy contained in the damping waves radiated away from the ship during each wave period, that is, the left part of the final expression for the calculation of the added resistance $R_{x}$ :

$$
\left(-R_{x}\right)(U-c) T_{e}=\frac{\pi}{\omega_{e}} \int_{L}\left(b_{33}-U \frac{d a_{33}}{d x}\right)\left|U_{R Z}\right|^{2} d x
$$

where $\omega_{e}$ and $T_{e}$ are encounter frequency and encounter period, $L$ is ship length, $\omega$ is the frequency of the incident wave, and $U_{R Z}$ is the vertical relative velocity of each ship section, $a_{33}$ and $b_{33}$ are two dimensional sectional added mass and damping coefficient of heave motion, respectively.

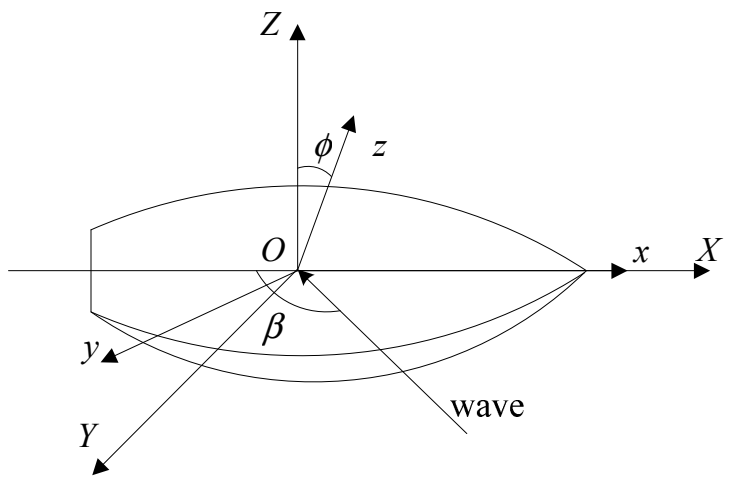

Fig. 1. Coordinate system

The calculation of added resistance by roll motion in oblique waves can be written. Similarly, within the strip theory approximation, the energy of encounter radiated due to roll motion at any time point is :

$$
P_{4}=\left(-R_{T r}\right)(-c-U \cos \beta)=\left(-R_{T r}\right) \frac{\lambda}{T_{e}}
$$

where $R_{T r}$ is the horizontal force (consequently has the direction of the incident wave propagation), $\lambda$ is the wave length, $c=g / \omega$ is the wave speed.

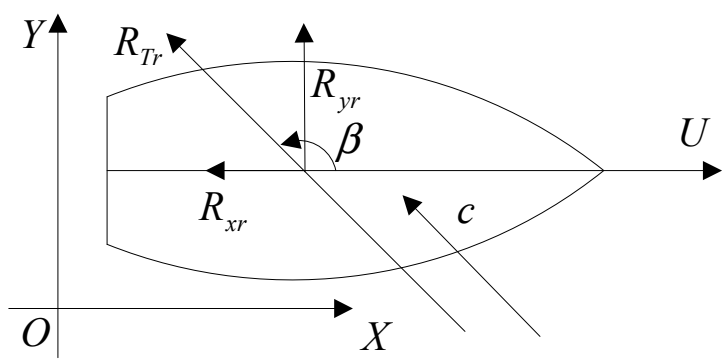

Fig. 2. Definition of added resistance and drift force

We simplify the roll motion damping coefficient of Loukakis and Sclavounos necessary for the calculation of the radiated energy is

$$
b_{4}=b_{44}-U \frac{d a_{44}}{d x}
$$


where $a_{44}$ and $b_{44}$ are two dimensional sectional added mass and damping coefficient of roll motion, respectively.

Therefore, the energy radiated by the roll motion at any time point is

$$
P_{4}=\int_{L} b_{4} \dot{\eta}_{4}^{2} d x
$$

The roll added resistance $R_{x r}$ and drift force $R_{y r}$ are the longitudinal and latitudinal components of the wave resistance $R_{T r}$, as illustrated in Fig. 2.

Hence, the final expressions, which allow us to calculate the time domain roll added resistance and drift force in oblique waves are:

$$
\begin{gathered}
R_{x r}=R_{T r} \cos (180-\beta)=-R_{T r} \cos \beta \\
R_{y r}=R_{T r} \sin (180-\beta)=R_{T r} \sin \beta
\end{gathered}
$$

\section{Speed loss calculation}

The effective power of a ship is the product of the total calm resistance of the naked hull and the nominal speed, therefore the effective power equation is

$$
P_{E}=R_{T S} U
$$

Assuming a ship is thrusted with constant power in rough waves, the effective power can be rewrited as

$$
P_{E}=\left(R_{T S}+R_{x r}\right) U_{0}
$$

So, the the actual forward speed $U_{0}$ can be obtained as follows:

$$
U_{0}=\frac{R_{T S}}{R_{T S}+R_{x r}} U
$$

The speed loss $\left(\Delta U=U-U_{0}\right)$ simulation results are presented in section .

\section{CONTROL System Design}

All ship motion control devices contain nonlinear dynamic parts because of their complicated hydrodynamic characteristics. For fins, the nonlinear term is a saturation element which is used for limiting rotate angles. The nonlinear generalized minimum variance (NGMV) [14] control scheme is to introduce the GMV controller for nonlinear processes using dynamic cost function weightings. The main benefits of the so called NGMV approach lie in the simplicity of the concepts and it has been applied successfully to ship rudder roll stabilization and yaw control systems [15][16]. Therefore, from the practical point of view, we choose the NGMV strategy.

\section{A. System description}

From Eqs. (4), (6) and (7), the value of roll added resistance is determined by the instantaneous roll rate value. The main control objectives are the reduction of roll motion and speed loss, so roll angle and roll rate must be controlled at the same time, are shown in Fig. 3.

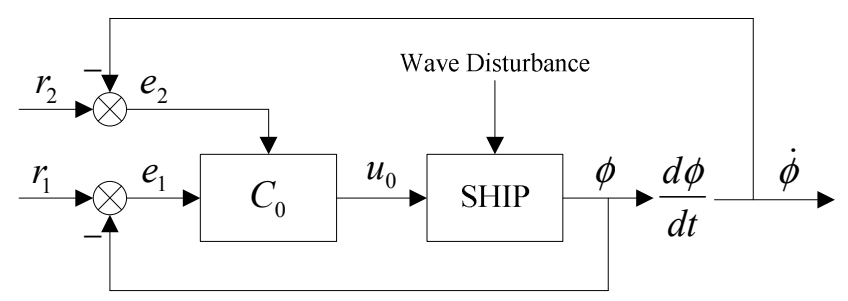

Fig. 3. The control system diagram

Actually, it is not a multivariable system as it looks, because there is only one kind of actuator-fins. Hence, the two variables must be controlled with two single controllers. The subscript 1 and 2 identity roll and roll rate components. $r$ represents the reference input signal and will be set to zero, $e$ denotes the error signal, $u_{0}$ is the total control signal. The double NGMV controller $C_{0}$ can be described in Fig. 4, where $C_{1}$ and $C_{2}$ are single NGMV controller for roll and roll rate respectively, the two weightings must satisfy:

$$
\left\{\begin{array}{l}
0 \leq \lambda_{1} \leq 1 \\
0 \leq \lambda_{2} \leq 1 \\
\lambda_{1}+\lambda_{2}=1
\end{array}\right.
$$

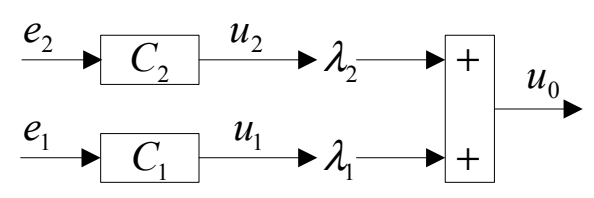

Fig. 4. The components of double NGMV controller

\section{B. System model}

We decouple linear roll equation from the other motion components which is excited by wave slope, describe as a second order polynomial transfer:

$$
G_{\phi}(s)=\frac{\omega_{n}^{2}}{s^{2}+2 \xi_{n} \omega_{n} s+\omega_{n}^{2}}
$$

where $\xi_{n}$ and $\omega_{n}$ are roll natural frequency and damping coefficient, respectively.

Linear wave response approximations are usually prefered by ship motion systems engineers, owing to their simplicity and applicability. A second order linear filter is adopted to fit the shape of ITTC double parameters spectrum. This model is written as:

$$
G_{w}(\mathrm{~s})=\frac{2 \xi_{w} \omega_{e} \sigma s}{s^{2}+2 \xi_{w} \omega_{e} s+\omega_{e}^{2}}
$$

where $\xi_{w}$ is the wave damping coefficient and $\sigma$ is a constant describing the wave intensity which determined by wave height and average period. 


\section{The NGMV algorithm} form:

A nonlinear plant model can be written in the following

$$
(\mathcal{W} u)(t)=z^{-k}\left(\mathcal{W}_{k} \mathrm{u}\right)(\mathrm{t})
$$

where $k$ denotes the plant time-delay, the nonlinear plant is separated into a linear part $W_{0 k}$ and a nonlinear part $\mathcal{W}_{1 k}$.

The plant itself is nonlinear and may have a quite general form (state-space, transfer operators, neural networks, etc.). However, the reference and disturbance signals are assumed to have linear time invariant (LTI) model representations. This is often valid, since in many appliactions the only models available for the disturbance and reference are LTI approximations. So, the two signals can be written as:

$$
d(\mathrm{t})=\mathrm{W}_{d} \delta(\mathrm{t})
$$

and

$$
r(\mathrm{t})=\mathrm{W}_{r} \zeta(\mathrm{t})
$$

where, for the anti-roll problem, the reference model is zero, $W_{d}$ represents the disturbance shaping filter, $\delta(\mathrm{t})$ and $\zeta(\mathrm{t})$ are white noise sources of unite variance.

The power spectrum for the combined reference and disturbance signal $f=r-d=Y_{f} \mathcal{E}$ can be computed as:

$$
\Phi_{f f}=\Phi_{r r}+\Phi_{d d}=W_{r} W_{r}^{*}+W_{d} W_{d}^{*}
$$

and the strictly minimum phase generalized spectral-factor $Y_{f}$ satisfies:

$$
Y_{f} Y_{f}^{*}=\Phi_{f f}
$$

here $*$ denotes the complex conjugate transpose operate, $\varepsilon(\mathrm{t})$ is a zero mean white noise and a measurment noise model has not been included to simply the equations.

The optimal NGMV control problem involves the minimization of the variance of the following signal:

$$
\phi_{0}(\mathrm{t})=\mathrm{P}_{c} e(\mathrm{t})+\left(\mathcal{F}_{c} u\right)(\mathrm{t})
$$

It has a dynamic cost function weighting $P_{c}\left(\mathrm{z}^{-1}\right)$ on the error signal, represented in linear polynomial form as: $P_{c}=P_{c d}^{-1} P_{c n}$. It also includes a nonlinear dynamic control signal cost operator term $\left(\mathcal{F}_{c} u\right)(\mathrm{t})$. Typically, $P_{c}$ is low-pass and $F_{c}$ is constant or a high-pass transfer. The signal $\phi_{0}(\mathrm{t})$ is to be minimized in a variance sense, so the cost function:

$$
J=E\left\{\phi_{0}^{2}(\mathrm{t})\right\}
$$

If the plant time-delay is of magnitude $k$, this implies the control at time $t$ affacts the output $k$ steps later. So the control signal costing can be defined as:

$$
\left(\mathcal{F}_{c} \mathrm{u}\right)(\mathrm{t})=\mathrm{z}^{-k}\left(\mathcal{F}_{c k} \mathrm{u}\right)(\mathrm{t})
$$

typically this will be a linear operator but it may also be nonlinear to compensate the plant input nonlinearities in appropriate cases. The delay free control weighting operator $\mathcal{F}_{c k}$ is assumed invertible.

Assuming the linear disturbance, reference and plant linear subsystem models have the left-coprime polynomial marix representation:

$$
\left[\mathrm{W}_{d}, \mathrm{~W}_{r}, \mathrm{~W}_{0 k}\right]=\mathrm{A}^{-1}\left[\mathrm{C}_{d}, \mathrm{E}_{r}, \mathrm{~B}_{0 k}\right]
$$

then the spectral factor $Y_{f}$ can be written in the polynomial matrix form as $Y_{f}=A^{-1} D_{f}$.

Introduce the left coprime matrices $A_{p}$ and $P_{c f}$ satisfy the following equation:

$$
P_{c n} A^{-1}=A_{p}^{-1} P_{c f}
$$

Then the signal $\phi_{0}(\mathrm{t})$ can be rewritten as:

$$
\phi_{0}=\left(\mathrm{A}_{p} \mathrm{P}_{c d}\right)^{-1} P_{c f} D_{f} \mathcal{E}-\left(\mathrm{P}_{c} \mathcal{W}-\mathcal{F}_{c}\right) \mathrm{u}
$$

Introduce the diophantine equation to expand the combined disturbance and reference model into two groups of terms:

$$
A_{p} P_{c d} F_{0}+z^{-k} G_{0}=P_{c f} D_{f}
$$

Substituting into Eq. (25):

$$
\phi_{0}=F_{0} \varepsilon+\left(\mathrm{A}_{p} \mathrm{P}_{c d}\right)^{-1} z^{-k} G_{0} \varepsilon-\left(\mathrm{P}_{c} \mathcal{W}-\mathcal{F}_{c}\right) \mathrm{u}
$$

The first term on the right of Eq. (27) is independent of the control term and the smallest variance is achieved when the remaining terms are zero. Therefore, the optimal control signal must satify :

$$
u(\mathrm{t})=\left(\mathrm{F}_{0} \mathrm{Y}_{f}^{-1} \mathcal{W}_{k}-\mathcal{F}_{c k}\right)^{-1}\left(\left(\mathrm{~A}_{p} \mathrm{P}_{c d}\right)^{-1} \mathrm{G}_{0} \mathrm{Y}_{f}^{-1} \mathrm{e}\right)(\mathrm{t})
$$

so the single NGMV controller structure may be presented by the Fig. 5. The choice of the weighting operators $P_{c}$ and $\mathcal{F}_{c k}$ is described in [13].

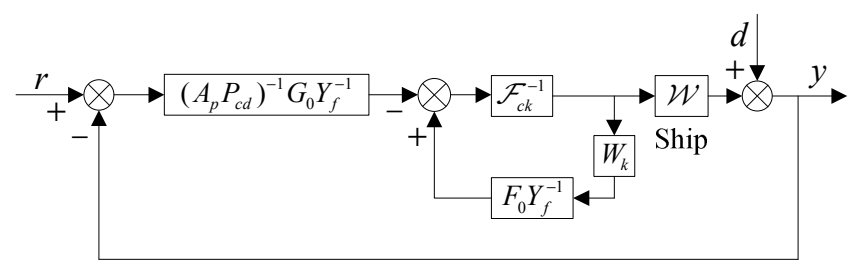

Fig. 5. Single NGMV controller structure

\section{SIMULATIONS}

The ship model introduced in reference [17] is adopted for calculations. It is a navy vessel with a design forward speed 15 kns and a magnitude constraint for the mechanical angle of the fins of $25^{\circ}$. Using a sea environment described by the ITTC spectrum parameterized with a wave height of $4 \mathrm{~m}$ and wave period of $7 \mathrm{~s}$ - top sea state 5. The classical PID controller was discretized and used as the basis of the NGMV design. Simulation results are shown in Fig. 6 and Fig. 7. 

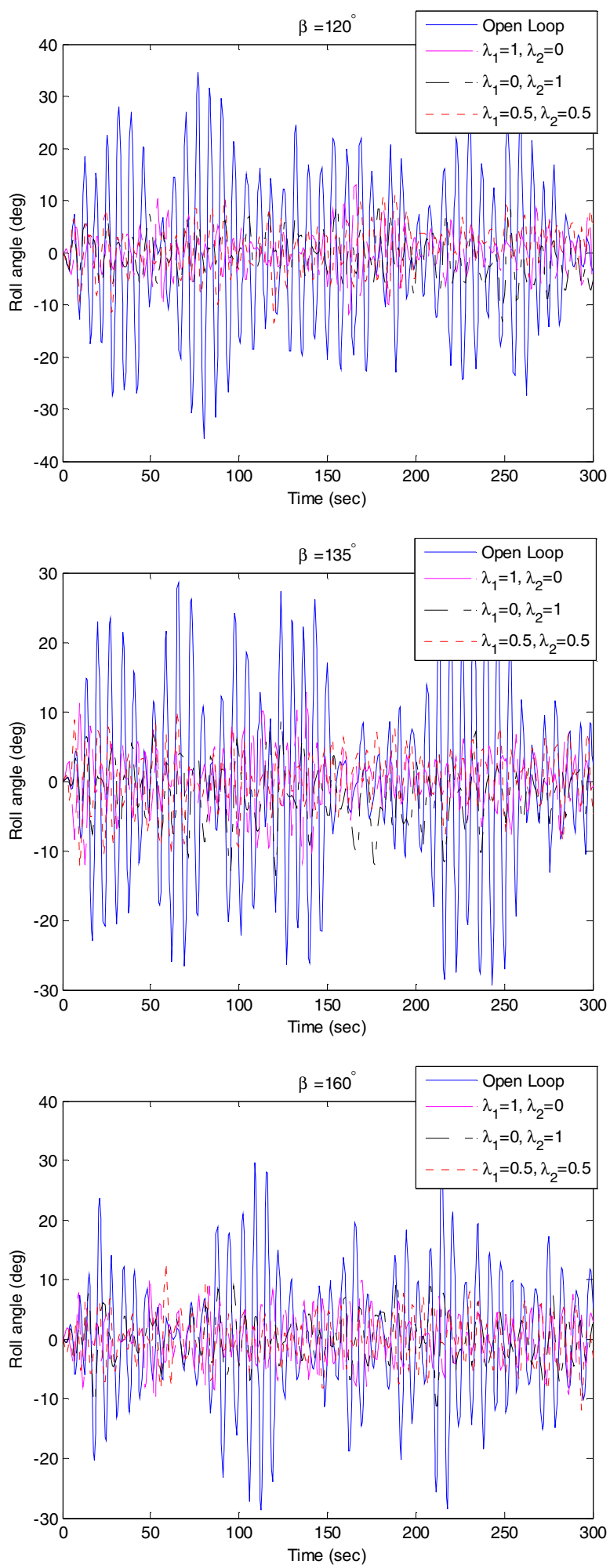

Fig. 6. Time trace of roll angle for different forward directions

In Fig. 6, time series of roll angle for three moving directions are displaced. We can appreciate that the three performance indexes have a similar anti-roll rate and fins work better with rough rolling parts. However, the magnitude of roll angle are increased at some time points for the latter two performance indexes, this may because of the demand of added resistance reduction. This phenonmenon does not affect the overall roll reduction effects. After all it is a statistics process and we may sure that the added resistance will not be the lowest with the smallest roll angle under the same requirement of roll reduction rate.
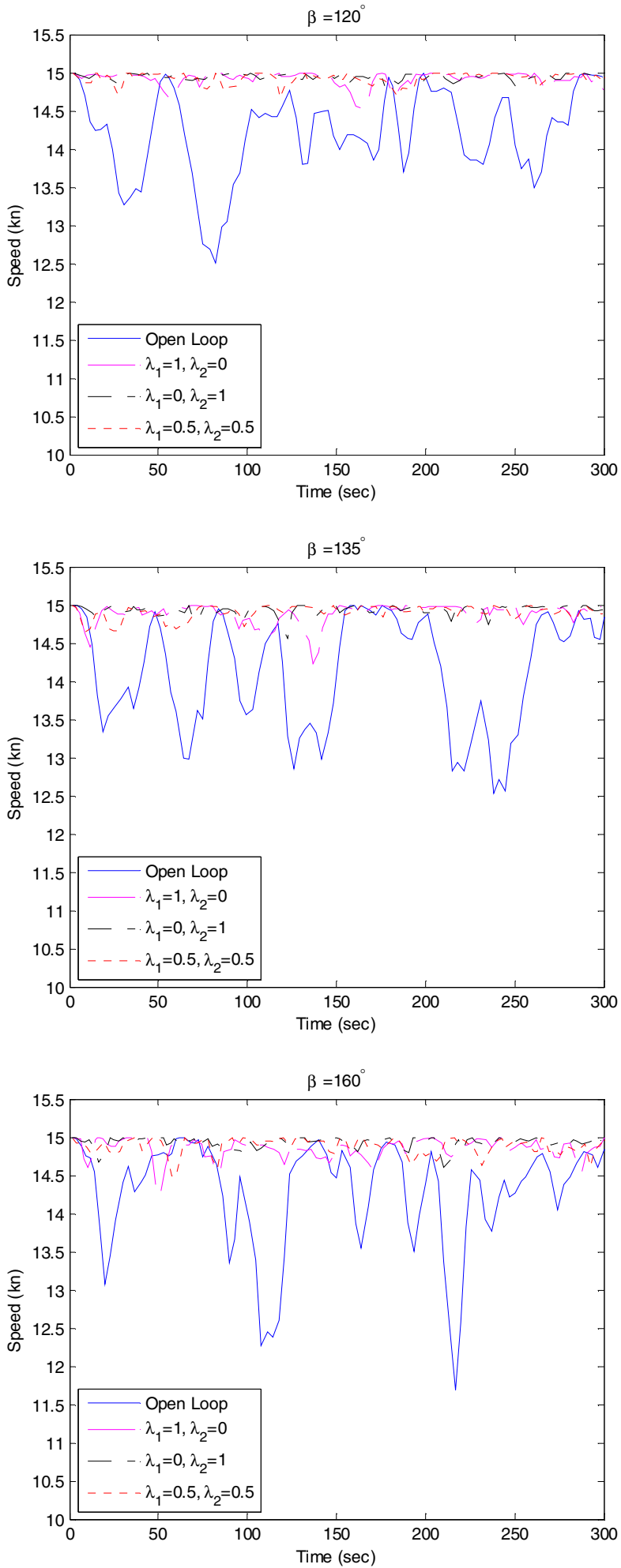

Fig. 7. Time trace of ship speed for different forward directions 
Fig. 7, shows a simulation with ship moving speed time series. The open loop average speed loss exceed $2 \mathrm{kns}$, it responses the conclusion in section that added resistande can be reduced by fins' actions. The speed can be maintained effectively by this roll stabilization control system.

TABle I. Performance of the Controller

\begin{tabular}{|c|c|c|c|}
\hline \multirow{2}{*}{ No. } & \multicolumn{3}{|c|}{ Comparison of Controlled and Uncontrolled } \\
\hline & Quantity & Value & Units \\
\hline \multicolumn{4}{|c|}{ Roll Reduction Percentage } \\
\hline 1 & Open loop (RMS) & 12.99 & deg \\
\hline 2 & $\lambda_{1}=1, \lambda_{2}=0$ & 68.07 & $\%$ \\
\hline 3 & $\lambda_{1}=0, \lambda_{2}=1$ & 68.10 & $\%$ \\
\hline 4 & $\lambda_{1}=0.5, \lambda_{2}=0.5$ & 68.97 & $\%$ \\
\hline \multicolumn{4}{|c|}{ Speed Loss Percentage } \\
\hline 5 & Open loop (AVE) & 12.54 & $\mathrm{kn}$ \\
\hline 6 & $\lambda_{1}=1, \lambda_{2}=0$ & 0.80 & $\%$ \\
\hline 7 & $\lambda_{1}=0, \lambda_{2}=1$ & 0.42 & $\%$ \\
\hline 8 & $\lambda_{1}=0.5, \lambda_{2}=0.5$ & 0.59 & $\%$ \\
\hline
\end{tabular}

The data of the table show the performance for the case $\beta=135^{\circ}$ depicted in Figs. 6 and 7. Obviously, the three performance indexes can achieve anti-roll objective in a similar degree. The value of average speed loss can over $2 \mathrm{kns}$ which is significant for the power of a moving ship, that is to say, we vertify the conclusion that the actions of fins can reduce added resistance and proper actions can achieve even more. We can see a higher performance of fin stabilizers when index $\lambda_{1}=0, \lambda_{2}=1$ is used. This maybe due to the expression of roll and roll rate:

$$
\begin{gathered}
\phi=\sum_{i=1}^{n} \phi_{u i} \sin \left(\omega_{i} t-\varepsilon_{i}\right) \\
\dot{\phi}=\sum_{i=1}^{n} \omega_{i} \phi_{u i} \cos \left(\omega_{i} t-\varepsilon_{i}\right)
\end{gathered}
$$

where the irregular wave was seperated into $n$ small regular waves, $\phi_{u i}$ and $\varepsilon_{i}$ are the amplitude and phase of each regular wave. Each regular roll rate equation has a $\omega_{i}$ times value of roll amplitude at any time point, though they have different phases. So, the roll angle can be reduced with the reduction of roll rate in whole process.

\section{CONCLUSION}

In this paper, we have revisited the effect of added resistance associated with ship roll motion. This effect can affect the fuel consumption of a ship significantly in medium to severe sea state. The most important issue is that it may lead to ship speed loss. Because of this, a way to prevent this effect is to design a comprehensive controller which reduces not only roll angle but also minimises roll rate (i.e. in order to restrict the added resistance). Using this approach, we have formulated the control problem using a double NGMV strategy. The simulation results presented indicate that a significant improvement in speed maintain performance can be obtained by the application of the proposed control algorithm. From a practical point of view, therefore, it may be necessary to combine with roll angular velocity control when a roll stabilization control system is designed.

\section{ACKNOWLEDGMENT}

We are grateful for the fund of the China Scholarship Council (CSC) on the joint PhD student project. I also like to appreciate the help of Industrial Control Centre, University of Strathclyde on control algorithm and the support of National Natural Science Foundation of China (51279039).

\section{REFERENCES}

[1] J. Prpić-Oršić, O. M. Faltinsen, "Estimation of ship speed loss and associated $\mathrm{CO}_{2}$ emissions in a sea way," Ocean Engineering, Vol. 44, pp. $1-10,2012$

[2] IMO, "Prevention of air from ships," Second GHG study, London, 2010.

[3] Z. Chuang, S. Steen, "Speed loss due to seakeeping and maneuvering in zigzag motion," Ocean Engineering, Vol. 48, pp. 38-46, 2012.

[4] Z. Chuang, S. Steen, "Speed loss of a vessel sailing in oblique waves," Ocean Engineering, Vol. 64, pp. 88-99, 2013.

[5] M. C. Fang, Z. Y. Lee, K. T. Huang, "A simple alternative approach to assess the effect of the above-water bow form on the ship added resistance," Ocean Engineering, Vol. 57, pp. 34-48, 2013.

[6] F. P. Arribas, "Some methods to obtain the added resistance of a ship advancing in waves," Ocean Engineering, Vol. 34, pp. 946-955, 2007.

[7] K. Graf, M. Pelz, V. Bertram, H. Söding, "Added resistance in seaways and its impact on yacht performance," The 18th Chesapeake Sailing Yacht Symposium, Annapolis, Maryland, pp. 1-13, March 2007.

[8] R. Bhattacharyya, Dynamics of marine vehicles, New York: Wiley, 1978, pp. 237-238.

[9] H. Jin, Z. Liu, "Investigation on added resistance model base on roll motion attitude," Engineering Mechanics, Vol. 30, pp. 73-77, 2013.

[10] H. Jin, Z. Liu, "Fin stabilizers control systems for ships with optimum added resistance," Journal of Huazhong University of Science and Technology (Natural Science Edition), Vol. 41, pp. 115-119, 2013.

[11] Z. Liu, H. Jin, "Extended radiated energy method and its application to a ship roll stabilisation control system," Ocean Engineering, Vol. 72, pp. 25-30, 2013.

[12] T. A. Loukakis, P. D. Sclavounos, "Some extensions of the classical approach to strip theory of ship motions, including the calculation of mean added forces and moments", Journal of Ship Research, Vol. 22, pp. $1-19,1978$.

[13] J. Gerritsma, W. Beukelman, "Analysis of the resistance increase in waves of a fast cago ship," International Shipbuilding Progress, pp. 285292, 1972.

[14] M. J. Grimble, "Design of generalized minimum variance controllers for nonlinear systems," International Journal of Control Automation and Systems, Vol. 4, pp. 281-292, 2006.

[15] P. Majecki, R. Katebi, M. J. Grimble, "Rudder roll stabilization with nonlinear GMV control," International Control Conference, 2006.

[16] M. J. Grimble, P. Majecki, "Polynomial approach to non-linear predictive generalize minimum variance control," IET Control Theory and Applications, Vol. 4, pp. 411-424, 2010.

[17] T. Perez, Ship motion control: Course keeping and roll reduction using rudder and fins, London: Springer, 2005, pp. 274-279. 